ISSN 1112-9867

Available online at

http://www.jfas.info

\title{
CONTRIBUTION TO THE STUDY OF THE BIODIVERSITY OF BENTHIC INVERTEBRATES AND THE BIOLOGICAL QUALITY OF SOME RIVERS IN THE WATERSHED BOUMERZOUG (EAST OF ALGERIA)
}

\author{
N. Bekhouche ${ }^{1}$, F. Marniche ${ }^{2}$ and A. Ouldjaoui ${ }^{*}$ \\ ${ }^{1}$ Université Larbi Ben M'hidi, Oum El Bouaghi. Algérie \\ ${ }^{2}$ Ecole Nationale Supérieure Vétérinaire d'El Alia, Alger, Algérie
}

Received: 27 May 2016 / Accepted: 27 December 2016 / Published online: 01 January 2017

\begin{abstract}
The composition of invertebrate communities colonizing the bottom of rivers, called for this reason benthic macro invertebrates, allows characterizing the biological quality of river water.This study focuses on benthic macrofauna of oued Boumerzoug and its two tributaries: Oued El Guareh and Oued Segus. Eleven (11) stations were prospecting object, between December 2014 and April 2015.The six sampling campaigns have identified a faunal population consisted of 7364 individuals, distributed in 106 taxa, with predominance of Diptera and the lack of sensitive polluo- elements (plécoptères). The results of analysis by method " IBGN " reveal poor hydro biological quality in the stations (G1, G2, G3, S1, S2, S3, S4, B1) and bad qualities for stations (B2, B3, B4).
\end{abstract}

Key words: Benthic macro-invertebrates ; Bio- indicators ; Pollution ; IBGN ; Quality.

Author Correspondence, e-mail: ouldjaoui33@yahoo.fr

doi: http://dx.doi.org/10.4314/jfas.v9i1.16

\section{INTRODUCTION}

La recherche en écologie sur les eaux courantes en Algérie étaient fragmentaire citons ceux de SEURAT (1922,1930), GAUTHIER (1928), et sur le milieu madicole, marginal, VAILLANT(1955). Actuellement, les programmes d'étude en hydrobiologie se développent rapidement dans ce pays par AIT MOULOUD, 1981, ARAB et ZARDI 1983, DJERIDANE et SAHLI 1983, GAGNEUR et COLL 1986, ALIANE 1986, GAGNEUR 1987 ; LOUNACI 
1987[1], LOUNCI-DAOUDI (1996), LOUNACI et al. (2000); MEBARKI (2001), LOUNACI (2005) ; LOUNACI et VINÇON (2005) et MOUBAYED-BREIL et al. (2007)[2]. Rappelons que, l'eau revêt en Algérie un caractère stratégique du fait de sa rareté et d'un cycle perturbé et déséquilibré [3]. De plus, les organismes aquatiques qui peuplent ces eaux, manifestent des préférences et des exigences vis-à-vis des différents facteurs biotique et abiotique : lorsqu'un changement survient dans un milieu aquatique, on constate des perturbations des communautés qui le peuplent. Parmi les communautés biologiques, les communautés de macro- invertébrés benthiques sont couramment utilisées pour évaluer l'état de santé global des écosystèmes aquatiques. HELLAWELL (1986); BARBOUR et al. (1999); Européen Commission (2005). Ce sont des organismes visibles à l'œil nu, tel que les insectes, les mollusques, les crustacés et les vers, qui habitent le fond des cours d'eau et les lacs. Ces organismes constituent un important maillon de la chaine alimentaire des milieux aquatiques. Ils sont reconnus pour être de bons indicateurs de la santé des écosystèmes aquatiques en raison de leur sédentarité, de leur cycle de vie varié, de leur grande diversité et de leur tolérance variable à la pollution et à la dégradation de l'habitat. Abondants dans la plupart des rivières, ils sont faciles à récolter et leur prélèvement a peu d'effets nuisibles sur le biote résident (BARBOUR et al. 1999) [4]. A les oueds (Guareh, Segus et Boumerzoug) situés au niveau sous bassin versant de Boumerzoug, ces invertébrés sont non connus et non étudies auparavant malgré les travaux menés dans quelques rivières voisines tels que Oued Rhumel [5,6].Le présent travail, mené durant six mois, sur trois oueds situés au niveau de sous bassin versant de Boumerzoug constitue une étude hydro- biologique qui se fixe pour objectif de :

- Dresser une liste faunistique des différents groupes systématiques.

- Evaluer la qualité des eaux de quelques cours d'eau de sous bassin de Boumerzoug à l'aide de l'indice biologique global normalisé (IBGN ; AFNOR ,1992) [7].

\section{METHODES D'ETUDE}

\subsection{Zone d'étude et stations de prélèvement}

\section{2 .1.1. Zone d'étude}

Les oueds étudiés appartiennent au bassin partiel de Boumerzoug qui est situé dans le nordest de l'Algérie. Il est considéré comme un bassin antérieur de Kabir El Remel, qui occupe une superficie totale de $1832 \mathrm{~km}^{2}$. Il représente une zone de transition entre le versant sud de l'Atlas Tellien et les Hautes plateaux, le bassin de Boumerzoug a pour coordonnées géographiques35, $53^{\circ}$ à $36,25^{\circ}$ d'altitude Nordet 6,28 à $7,4^{\circ}$ longitudes Est. 
Ce dernier est situé au nord par le khroub, au sud et au sud-est par le tell d'ainKircheetà l'Est par Ain Abid et le tell de Sigus, à l'Ouest par El Guerah et au Sud-Ouest par le tell d'AinMlila. Le oued Boumerzoug est considéré comme le principale oued du Bassin, son caractéristique essentiel est basé sur sa sinueuse et de rebondissements tous au long de son trajectoire. Il ressort dès l'OuyounBomerzoug appelé "Ain Mlila"ensuite il prend la direction sud-ouest d'oued Fourchi vers le Nord et le Nord-Est. Sa longueur est de 31,5km, il est constitué de : Bassin partiel de Ain Mlila, le bassin partiel de Sigus vers Ain fakroun, le bassin partiel de Bounouara vers Ain Abid qui sont oued El Berda, Oued El Klab, Oued El Maleh (oued Feskia). L'oued El Klab comprend un bassin partiel de Sigus, il prend sa source dans l'oued deKlab à une altitude de $800 \mathrm{~m}$ sur les configurations Myocène, sur la rive droite répond à certains récifs qui pénètrent formations Marinien sur le haut Sigus, soit à droite se nourrit à partir du versant nord du mont Gotas, cette dernière se rencontre avec l'oued El Malehet forme l'oued Bomerzoug. L'oued Sigus représente un cours d'eau très important pour la région de Sigus, a une longueur de 4,77 $\mathrm{km}$ de territoire communale. Mais la partie qui coupe la longueur de la ville est de $2,35 \mathrm{~km}$ qui se retrouve dans les limites de la ville (Direction de la construction et de la reconstruction d'Oum El Bouaghi,2015).l'oued El Guareh prend sa source au niveau de haut de la source El Feskia aux pieds de l'ouest du mont Keryoun, se réunie à la rive gauche de ouedForchi avant sa confluence avec ouedSigusil ce ramifiepar certains des coraux, qui vient de la pente sud du mont Tikbab et le versant nord du mont el Birmaavec des déviation moyenne à partir de ça vont former oued El Malehqui bifurque de l'oued El Guareh.

\subsubsection{Stations de prélèvement}

Le choix de ces stations a été effectué en tenant compte de certaines paramètres tel que l'altitude, la pente, la diversité des biotopes, l'amont et l'aval des agglomérations afin d'estimer l'importance de l'impact humain, et dans une certaine mesure, la régularité de la répartition des stations le long des cours d'eau. Ainsi que le conditionnement de l'accessibilité aux stations. Onze stations ont été retenues, et ont fait l'objet d'échantillonnage mensuel durant la période allant de Décembre 2014 à Avril 2015. La figure $n^{\circ} 1$ donne les situations des onze stations étudiées à savoir :

Oued El Guareh: 3 stations représentées par G1, G2 et G3.

Les trois stations sont situées en aval de la commune d'Ain M'lila. Elles permettent de mettre en évidence l'impacte des rejets industriels et urbains de la commune d'Ain M'lila.

Oued Segus: 4stations représentées par S1, S2, S3 et S4. 
Les quatre stations sont situées en amont de la commune de l'ouledRahmone et de la confluenceSegus-Boumerzoug.Ces stations rendent compte de la qualité des eaux d'oued Boumerzoug.Elle permet de mettre en évidence l'impact des rejets urbains de la commune d'ouledRahmoune et commune de Segus .

Oued Boumerzoug : 4stations présentées par B1, B2, B3 et B4.

B1 : Cette station est située en aval de la commune d'ouledRahmoune et en aval de la confluence El Guareh-segus -Boumerzoug. Cette station permet de mettre en évidence l'impact des rejets urbains de la commune de l'ouledRahmoune et rend compte de la station après le mélange des eaux des trois oueds.

B2 : Cette station est située en aval de la commune d'El Khroub été en amont de la Confluence Boumerzoug-Hamimine. Cette station traduit la qualité des eaux de l'oued Boumerezougsoumi aux rejets industriels et urbains d'El Khroub.

B3 : Cette station est située en aval de la confluence Hamimine -Boumerzoug et en amont de la zone industrielle Boumerzoug. Elle rend compte de la situation après le mélange des deux oueds.

B4 : Cette station est située en aval de la zone industrielle Boumerzoug et en amont de la confluence Boumerzoug - Rhumel. 


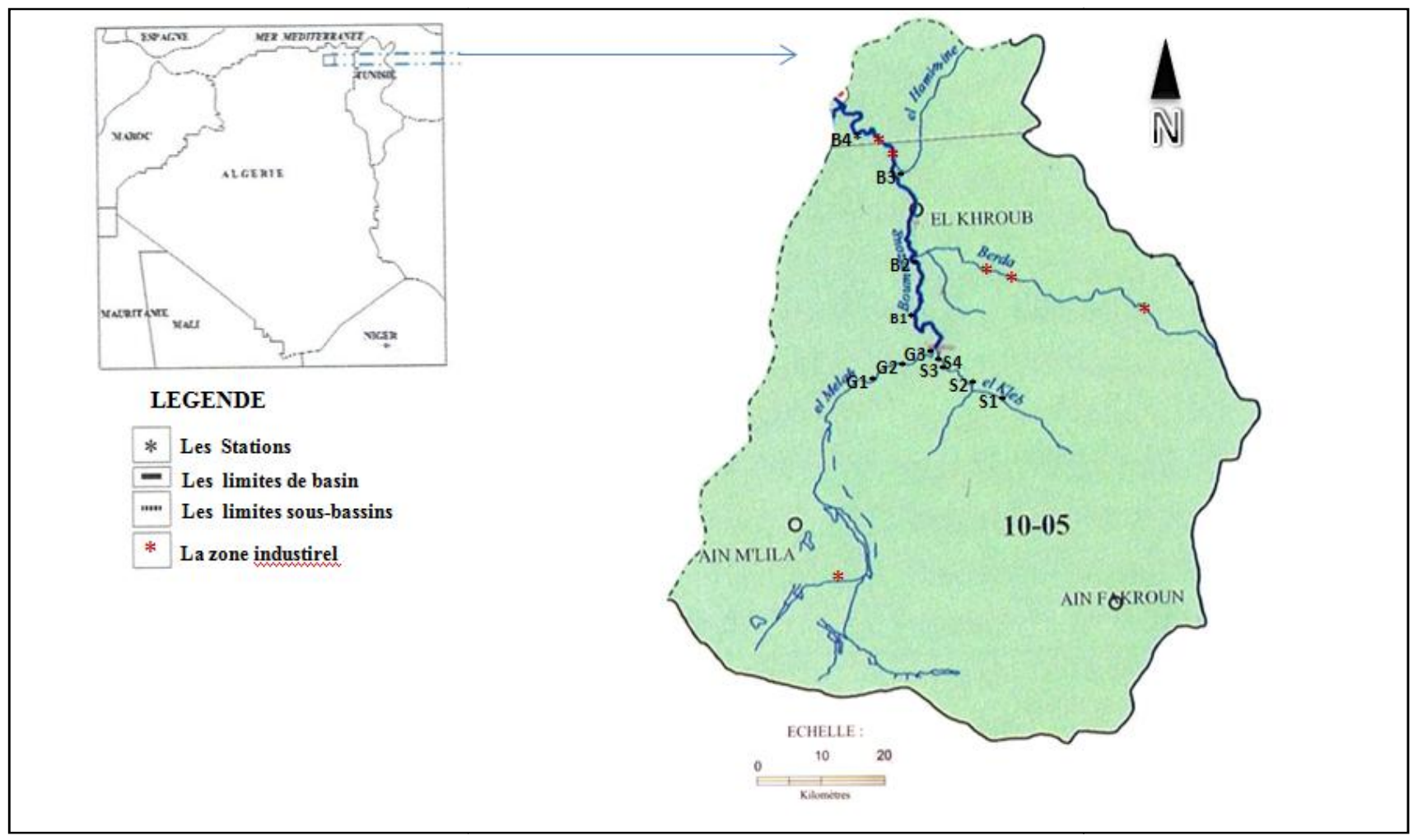

Fig.1. Cours d'eau étudiés et emplacement des stations(A.B.H.2002) [8]

\subsection{Techniques d'échantillonnage}

Le potentiel biologique est limite par la qualité de l'habitat physique formant la trame sur laquelle les communautés biologiques se développent [9]. Les choix de l'emplacement des points de prélèvement est fait en fonction de l'objectif de l'étude. Pour cela, on sélectionne en général un tronçon de cours d'eau dont la longueur est sensiblement égale à $50 \mathrm{~m}$, ou bien qui représente approximativement dix fois la largeur du lit mouillé nommé la station qui est l'unité de base de l'échantillonnage [10]. La première étape consiste à remplir la fiche de terrain répertoriant toutes les caractéristiques du cours d'eau, mais aussi de son environnement tels que les caractéristiques environnementales, l'identification de la station (nom et localisation), les caractéristiques du lit (largeur, facies d'écoulement, nature des berges et végétation des rives), les caractéristiques du fond (granulométrie et nature des substrats) et enfin la description de la végétation aquatiques. L'échantillonnage consiste diversité à rassembler la plus grande faunistique représentative des habitats à étudier pour obtenir un bilan plus complet possible des taxons présents dans les cours d'eau. L'indice comporte 8 prélèvements benthiques. Pour cela, on a choisis les couples substrat par vitesse les plus représentatifs, et biogènes. Si une station ne pas 8 types de supports différents, le nombre de prélèvements est complété à 8 par des prospections réalisées sur le support dominant mais pour des vitesses différentes. 


\subsection{Matériels (échantillonnage benthique)}

Le matériel biologique provient de prélèvements benthiques. Ils ont été effectués à laide d'un filet Suber pour le facies l'otiques et d'un filet troubleau pour le facies lentisques.

Milieu l'otique : les prélèvements de la faune sont effectués sur surfaces de l'ordre du $1 / 20(25 \mathrm{~cm} 20 \mathrm{~cm})$.Ils sont réalisés dans des zones peu profondes inférieure à $40 \mathrm{~cm}$.

Pour chaque récolte, l'opérateur a été le même, de façon à mainteneur les conditions de prélèvements aussi voisine que possible d'une série à l'autre.

L'échantillonnage de type «Surber »composé d'un filet à maille de $500 \mu \mathrm{m}$, et d'un cadre métallique, il est utilisé pour les milieux d'courantes (faciès l'otique : là où la vitesse de l'eau est rapide)[11].Ils sont placés sur le substrat que l'on souhaité échantillonné, ouverture du filet face au courant .Le substrat dur est frotté à la main de manière à décrocher les organismes qui sont alors entrainés par le courant dans le filet (pour les substrats meubles, l'échantillonnage se fait sur une épaisseur de quelques centimètres). On parle alors d'un échantillonnage quantitatif (surface échantillonnée identique quel que soit le prélèvement), c'est -à-dire que l'on peut rapporter les résultats à une surfaces de substrat.

Milieu lentisque : dans les zones d'eau calme ou se dépose les sédiments fins, lesprélèvements ont été réalisés à l'aide d'un filet troubleau (filet à manche) à ouverture circulaire de $30 \mathrm{~cm}$ de diamètre. L'échantillonnage est réalisé par dragage au filet des fonds sablonneux limoneux et/ou vaseux en Faisant des mouvements de va et vient sur une distance d'un mètre environ.

\subsection{Conservation des échantillons}

Les échantillons récoltés sont transfères dans un récipient étiqueté en précisant le lieu du prélèvement, la date et les caractéristiques du site .L'échantillon est conservé au formol à $5 \%$ dans le cas où la détermination ne s'effectue pas immédiatement après le prélèvement.

\subsection{Tri et détermination}

Cette opération consiste à extraire la faune du substrat contenu dans l'échantillon. Elle se fait au laboratoire, ou les échantillons sont rincés sur une série de tamis de mailles de tailles décroissante ( 5 à $0,2 \mathrm{~mm}$ ) afin d'éliminer au maximum le substrat fin restant et les éléments grossiers (graviers, plantes et feuilles). Le contenu des tamis est ensuite versé dans une bassine puis transvasé dans des tubes contenant de l'éthanol à $70 \%$. Un pré-tri et une identification de faune est faite sous la loupe binoculaire. Les organismes sont manipules à l'aide de pinces fine dans des boites à pétri à fond quadrillé. Les déterminations des 
spécimens récoltés sont réalisées en faisant appel à des ouvrages, collections et clés de détermination des macro- invertébrés [12].

\subsection{Analyse de la structure du peuplement par l'étude de l'indice IBGN}

L'indice Biologique Générale Normalisé (I, B, N, G) est déterminé grâce aux relevés des invertébrés aquatiques et des vertébrés tels que les insectes aquatiques et terrestres, nymphes et larve des diptères, les vers, les mollusques et les batraciens présents sur un tronçon de cours d'eau. Pour chaque station, l'échantillon d'invertébrés est constitué de prélèvements effectués séparément dans des habitats distincts.

\section{RESULTATS ET DISCUTIONS}

Les résultats vont porter sur l'inventaire des macro-invertébrés, sur l'analyse globale de la faune Abondance de la faune benthique et enfin l'analyse de la structure de peuplement par le calcul de l'indice biologique Global Normalise (IBGN).

\subsection{Inventaire des macro- invertébrés}

L'inventaire faunistique établi (liste ci-dessous), regroupe la répartition des peuplements dans les différentes stations d'échantillonnage :

Classe : CLITELLATA

Ordre : HAPLOTAXIDA

Famille: Tubificida

Genre : Tubifex

Tubifexsp. (Lamarck, 1816)

Tubifex tubifex

Ordre : OPISTHOPORA

Famille:Lumbricidae

Genre : Eiseniella

Eiseniellatetraedra

Genre : Satchellius

Satchelliussp.

Ordre : ARCHYNCHOBDELLIDA

Famille : Erpobdellidae

Genre : Dina

Dina parva

Classe : GASTROPODA

Ordre : PULMONAE
Famille :Helicidae

Genre : Eobania

Eobaniavermiculata

Famille :Sphincterochilidae

Genre : Sphincterochila

Sphincterochilacandidissima

Famille :Physidae

Genre :Physa

Physasp.

Genre : Aplexa

Aplexasp.

Genre :Physella

Physellagyrina

Famille : Hygromiidae

Genre : Cochlicella 
Cochlicellabarbara

Famille :Sublinidae

Genre : Rumina

Rumina decollata

Famille : Lymnaeidae

Genre : Lymnaeidae

Lymnaeidaesp.

Famille :Marginellidae

Genre : Marginellidae

Marginellidaesp.

Famille :Hydrobiidae

Genre :Lyogyrus

Lyogyrussp.

Genre :Heleobia

Heleobiasp.

Classe : OSTRACODA

Ordre : PODOCPIDA

Famille : Candonidae

Genre :Candona

Candonacandia

Classe : MALACOSTRACA

Ordre : AMPHIPODA

Famille :Gammaridae

Genre : Gammaridea

Gammarideasp.

Genre : Gammarus

Gammarussp .

Classe : INSECTA

Ordre : ODONATOPTERA (Zygoptera)

Famille : Caenagrionidae

Genre : Caenagrionidae

Caenagrionidaesp. (Larve)

Genre : Enallagma

Enallagmasp. (Larves)
Genre : Caenagrion

Caenagrionsp. (Larves)

Famille : Libellulidae

Genre : Orthetrum (Larve)

Orthetrumsp. (Larve)

Ordre : DIPTERA

Famille :Dolichopodidae

Genre : Dolichopodidae

Dolichopodidaesp. (Larve)

Genre : Rhaphilum

Rhaphilumsp.

Famille :Psychodidae

Genre : Psychoda

Psychodasp. (Nymphe)

Psychodasp. (Larve)

Psychodasp. (Adulte)

Psychodaalternata (Nymphe)

Genre : Clogmia

Clogmiasp. (Nymphe)

Clogmiasp. (Larve)

Genre :Pericoma

Pericomasp. (Larve)

Famille : Chironomidae

Genre : Chironomalidae

Chironomalidaesp. (Nymphe)

Genre : Chironomus

Chironomussp. (Larves)

Chironomussp. (Pupes)

Chironomussp. (Adulte)

Genre : Paratendipes

Paratendipessp.

Famille :Simuliidae

Genre : Simulium

Simuliumsp. 
Simuliumsp. (Nymphe)

Famille : Hydrophilide

Genre : Cercyon

Cercyonsp. (2 elytres)

Famille : Ephydridae

Genre : Ephydridae

Ephydridaesp. (Adulte)

Ephydridaesp.

Famille : Sciaridae

Genre : Bradysia

Bradysiasp.

Famille : Clucidae

Genre : Culex

Culexsp. (Nymphe)

Famille :Sphaeroceridae

Genre : Leptocera

Leptocerasp. (Adulte)

Famille :Cecidomyiidae

Genre : Cecidomyiidae

Cecidomyiidaesp. (Larve)

Famille : Syrphidae

Genre: Scaeva

Scaevapyrastri (Adulte)

Genre : Ecistalis

Ecistalistenax (Larve)

Famille :Tipulidae

Genre : Tipula

Tipulasp. (Larve)

Famille :Stratiomyidae

Espèce : Stratiomyidaesp.

Famille :Muxidae

Espece :Muxidaesp.

Famille :Phoridae

Genre : Aphiochaeta
Aphiochaetasp.

Famille : Opomyzidae

Genre : Opomyza

Opomyzasp.

Famille :Empididae

Genre : Empis

Empissp.

Famille : Bionidae

Genre : Dilophus

Dilophussp.

Famille :Chloropidae

Genre : Chlorops

Chloropssp.

Famille :Anthomyidae

Genre : Limnophora

Limnophorariparia (larve)

Ordre : COLEOPTERA

Famille :Staphylinidae

Genre : Philontus

Philontussp.

Genre : Atheta

Athetasp.

Genre :Plastystethus

Plastystethusabutaceus

Genre :Aleochara

Aleocharasp.

Genre : Medon

Medonsp.

Famille :Chrysomelidae

Genre : Phylloreta

Phyllotretaprocera

Genre : Aphthona

Aphthonamonstriata

Genre : Cassida 
Cassida( Cassidulella) vittata

Genre : Timarcha

Timarchasp.

Genre : Oulema

Oulemamelanopus

Famille :Dytiscidae

Genre : Laccophilus

Laccophilushyalinus

Genre : Hygrobiidae

Famille : Curculionidae

Especes :Curculionidae (Elytres)

Genre : Magdalis

Magdalissp.

Genre : Sitona

Sitonasp.

Famille : Carabidae

Genre : Tricholaemus

Tricholaemussp.

Genre : Microlestes

Microlestessp.

Genre : Ophonus

Ophonussp.

Genre :Microlestes

Microlestescorticalis

Famille :Haliplidae

Genre : Haliplus

Haliplussp.

Famille :Scarabeidae

Genre :Rhizotrogus

Rhizotrogussp.

Famille : Noteridae

Genre : Noterus

Noterussp.

Famille :Bruchidae
Genre : Bruchidius

Bruchidiussp.

Genre : Platytethus

Platytethusalutaceus (male)

Famille :Coccenellidae

Genre : Platynaspis

Platynaspisluteorubra

Ordre : HYMENOPTERA

Famille :Fourmicidae

Genre : Tapinoma

Tapinomasimrothi

Tapinomasp.

Genre : Messor

Messorsp.

Messorbarbarus

Genre : Dorylus

Dorylusfulvus

Genre : Cataglyphis

Cataglyphisviaticus

Genre : Plagiolepis

Plagiolepisbarbarus

Genre :Monomorium

Monomoriumsp.

Genre :Componotus

Componotussp.

Genre : Crematogaster

Crematogastersp.

Genre :Pheidole

Pheidolepallidala

Genre :Tetramorium

Tetramoriumbiskrens

Famille :Figitidae

Genre :Kleidotoma

Kleidotomasp. 
Famille :Diapriidae

Genre : Trichopria

Trichopria(Ashmeadopria)sp.

Famille :Braconidae

Genre : Braconidae

Braconidaesp.

Famille :Ichneumonidae

Genre : Diadegma

Diadegmasp.

Ordre : EPHEMEROPTERA

Famille :Baetidae

Genre : Baetis

Baetissp.

Famille :Caenidae

Genre :Caenis

Caenissp.

Ordre : HOMOPTERA

Famille : Cixidae

Genre : Oliarus

Oliarussp.

Ordre : HEMIPTERA

Famille : Pyrrhocoridae

Genre: Pyrrhocoris

Pyrrhocoris apterus

Ordre : DERMAPTERA

Famille : Lubiduridae

Genre : Anisolabis

Anisolabissp.

Classe : TUBULINEA

Ordre : ARCELLINIDA

Famille : Arcellidae

Genre :Arcella

Arcellasp.

Classe : HIRUDINE
Ordre : RHYNCHODBELLIFORMES

Famille :Glossiphoniidae

Genre : Haementeria

Haementeriasp.

Ordre : ARHYNCHOBDELLIDA

Famille :Erpobdellidae

Genre : Dina

Dina parva

Classe : ARACHNIDA

Ordre : ARANEA

Famille : Lycisidae

Genre : Lycisidae

Lycisidae sp.

Famille : Gnaphosidae

Genre : Gnaphosidae

Gnaphosidae sp.

Classe: NEMATOMORPHA

Ordre: NEMATOMORPHA

Famille :Nematomorpha

Espèce : Nematomorpha

Classe : OSTRACODA

Ordre : PODOCOPIDA

Famille :Candonidae

Genre : Cryptocandona

Cryptocandonasp.

Classe : BRANCHIOPODA

Ordre : DIPLOSTRACA

Famille :Chydoridae.

Genre : Chydorus

Chydorusphaericus

Famille :Daphniidae

Genre : Daphnia

Daphniasp. (Eufs) 


\subsection{Analyse globale de la faune}

Les prélèvements effectués au cours des 6 compagnes de Décombre 2014 jusqu'à Avril 2015 ont parmi de recensés un totale de 7364 individus répartis en 106 taxons correspondant à 11 classes et à 64 familles. Ces abondances fluctuent suivant les stations, allant de 10individus à 2472 individus (Fig. 3).Ces variations spatiales pourraient être attribuées aux diverses influences que subit les milieux et aussi à la nature des différents habitats. En effet, un cours d'eau perturbé peut créer des conditions défavorables pour certains organismes (polluons-sensible) laissant la place ainsi à d'autres organismes plus tolérants (polluonsrésistants).

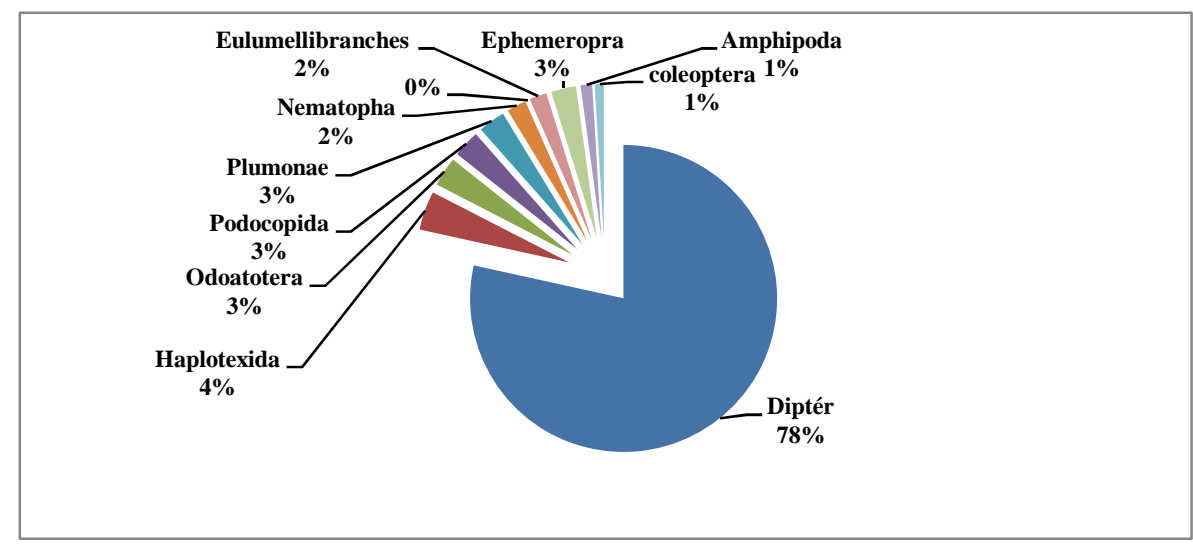

Fig.2. Abondance de la faune globale dans les eaux des stations étudiées.

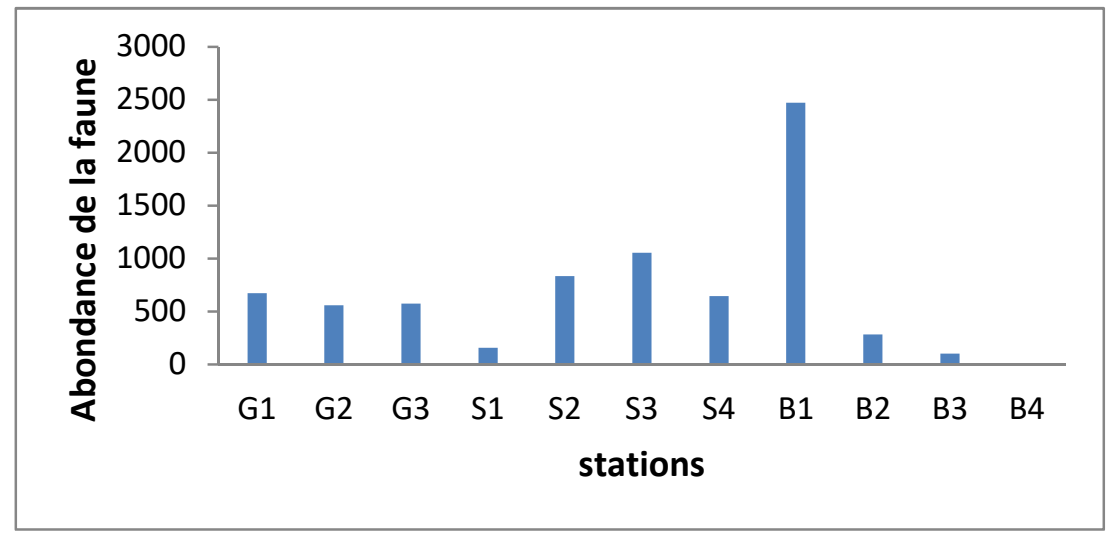

Fig. 3. Distribution de la faune globale dans les eaux des stations étudiées.

Sur 106 taxa d'invertébrées benthique, 78 taxa (soit 73,58\%) sont des insectes et 28 taxa (soit 26,41\%) appartiennent aux autre classe: Gastropoda, Clitellata, Ostracoda, Malacostraca, Tubulinea, Hirudine, Arachnida, Nematomorpha, Ostracoda, Branchiopoda, Bivalva. L'effectif du peuplement benthique montre que les Diptères sont nettement dominants (Fig.3). 
Il représentante 78,38\% (soit 5905 individus à 20 familles) de lafaune totale. Ils sont abondants dans la plupart stations. Pour les autres catégories sont faiblement représentés. Les peuplements de macro-invertébrés récoltés dans les trois oueds sont marqués par l'absence totale des plécoptères. Ce groupe connu par sa forte polluons-sensibilité est inexistant ; ceci laisse supposer au vu de nos prélèvements soit l'existence d'une pollution d'origine affluente qui est un facteur limitant de vie pour ce groupe ; soit les exigences écologiques de ce groupe (la température, l'oxygène dissous, la végétationbordant et la nature du substrat).En outre les fortes teneurs en calcium et magnésium (dureté) Peuvent également selon [13].

\subsection{Abondance de la faune benthique}

La totalité des macro-invertébrés capturés, triés, identifiés dans nos analyses est de 7364 invertébrés. L'abondance de chaque taxon fluctue suivant les stations. Elles varient de 10 individus à 2472 individus (Fig.3).Les sites affectés par une légère pollution organique ont abondance importante de la présence des deux familles de Diptères tels que les Chironomides et les Simuliides qui supportent la pollution organique. En effet, les stations (B1, S3) ont des abondances variant de 1053 individus (S3) à 2472individus (B1). Les stations (G1, G2, G 3, S1, S2) ont une structure assez équilibrée avec des abondances qui varient de 156 individus (station S1) à 833 individus (station S2). Ainsi, les stations d'aval (B3, B4) d'oued Boumerzoug qui est le plus affectés par la pollution urbaine ou industrielles, ils ont une structure déséquilibrée avec une abondance moins importante variant entre 10individus (B4) et 114 individus (station B3). En effet, les perturbations observées dans les secteurs de ces stations se traduisent par une modification dans les substrats, et plus celui-ci s'uniforme, plus le nombre de niche écologiques ne diminuent suivi d'une réduction du nombre d'espèces adaptées à ce type de milieu.

\subsubsection{Analyse qualitative et quantitative de la faune benthique}

\section{* Diptères}

Le matériel biologique récolté est compos é de larve, de nymphes et d'adultes. 5.704 individus appartenant à 20 familles ont été dénombrés dans l'ensemble des stations étudiées (Fig. 4). Il représente $77,45 \%$ de la faune totale. Ils sont abondants dans la plupart des stations mais leur répartition est hétérogène. LesDiptères sont des taxons à large valence écologique, elles sont polluo-résistantes, eurytope, et supportent bien l'élévation de températures. Parmi les différentes familles, les Simuliidaes abondent dans les milieux riches en matières organiques, facteurs favorables à la prolifération des stades immatures [14]. Les familles rencontrées dans ce groupe sont d'importance inégale. Les Chironomidae, avec 4.749 individus, soit $83,25 \%$ des Diptères et $64,48 \%$ de la faune totale. Les sites des essaims chez 
les Chironomidae sont très variés. Ainsi, ils se regroupent soit très proche de la végétation à 0,5 et $2 \mathrm{~m}$ ou plus haut au sommet des arbres. Chez la plupart des espèces méditerranéennes la reproduction a eu lieu pendant toute l'année avec un ou plusieurs maximums d'intensité en fonction des saisons [15]. Les Chironomidae sont très répondus dans les cours d'eau étudiés, tant dans les stations haute que dans les stations de basse altitude (Fig.4). Les Psychodidae occupent la seconde place dans l'ordre des Diptères, ils constituent 9,81\% (560 individus) de ce peuplement et $7,60 \%$ de la faune totale. Dans la nature les habitats primitifs des psychodidae sont représentés par divers biotopes tels que les cavernes, les crevasses de rochers, les terriers de rongeurs, les creux dans les arbres et autres de ce genre. Les psychodidae sont généralement disséminés dans les régions de base et moyenne altitude qui correspondent à des conditions [16]. Parmilesrécolter on a retrouvé les Sciaridae (160 Individus), les Similiidae (69 Individus), les Ephydridae (48individus), les Culicidae (20 individus), les Tipulidae (18individus), les Sphaeroceridae et les Empididaereprésentent pour chacune (17individus).Pour la famille des Similiidae l'espèce Simuliumsp.en font des vecteur de maladies autant chez les humains que chez les animaux.Parmi ces maladies, l'onchocercose, une maladie causée par un parasite entrainant la cécité, en est un exemple chez l'humain. Des réactions allergiques et la fièvre de la mouche noire transmettent la leucocytozoonose, une maladie mortelle pour les dindes, les canards et les poulets [17]. Dans nos prélèvements les autres familles de Diptères ont une faible fréquence. Elles ne sont représentées que par (46individus), soit $0,80 \%$ du totale des Diptères. La distribution longitudinale de ce groupe d'insectes le long des cours d'eau étudiés. La plus grande richesse est aux stations G1,G2,G3 ,S1,S2,S3 et S4 avec respectivement 14,13,8,8,7,6 et 6taxons. Dans ces stations, caractérisées par un courant moyen à modéré, des températures relativement élevées, un substrat hétérogène et une pollution organique légère. Qui pourrait être favorable à la prolifération des invertébrés polluo-résistants. 


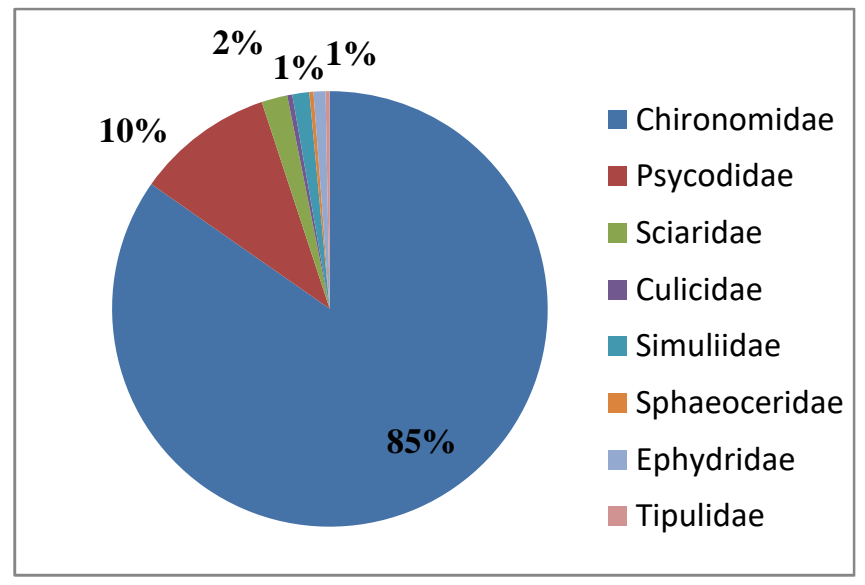

(A)

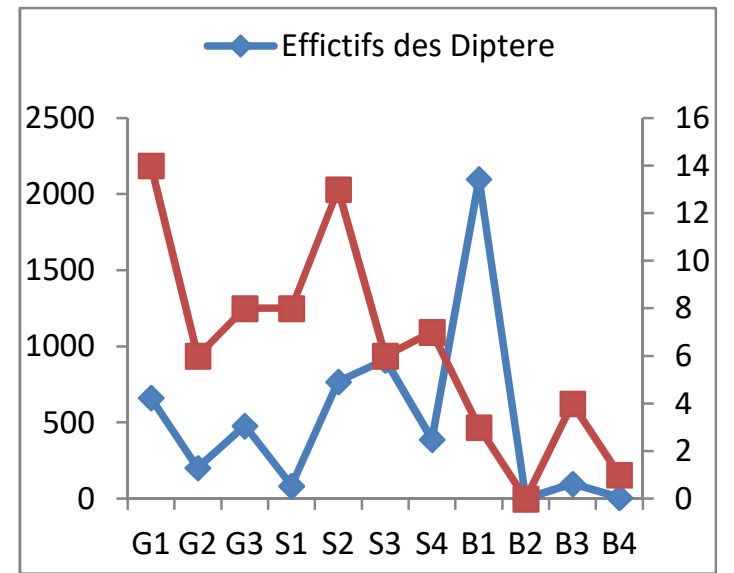

(B)

Fig.4. Abondance (A) et répartition longitudinale (B) des Diptères

\section{Haplotexida}

D'après, nos résultats, les Haplotexida sont représentés dans sous bassin versant de Boumerzougpar 301 individus, soit 4,08\% de la faune récoltée. Ils sont généralement représentés par la seule famille de Tubificidae et par un seul genre Tubifex (Fig.5). Représente le développement longitudinal des Haplotexida le long des trois oueds, elle montre que ces invertébrés se développement favorablement dans les stations situées en aval (G2, G3, S2, S3 et S4).Ce résultat est vraisemblablement lié à la présence des matières organiques, facteur favorable à la prolifération de ces organismes. Dans ces cinq stations, les Tubifex tubifex sont les plus dominants, avec un pic maximal en G2 .Cette capacité à résister à des températures élevées, de faibles concentrations en oxygène et des salinités élevées explique pourquoi certains espèces, notamment Tubifextubifex, sont particulièrement résistantes à la pollution organique. Cependant, la majorité des espèces vivent dans des habitats spécifiques et ne peuvent être réduites à ces formes polluo-résistantes [18]. 


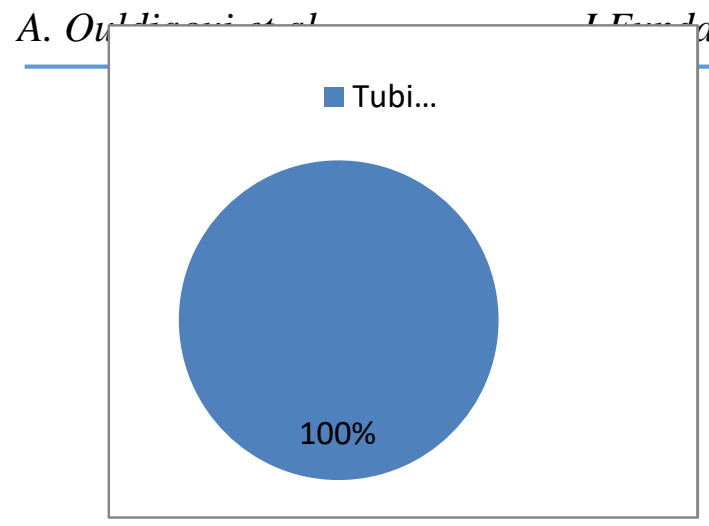

(A)

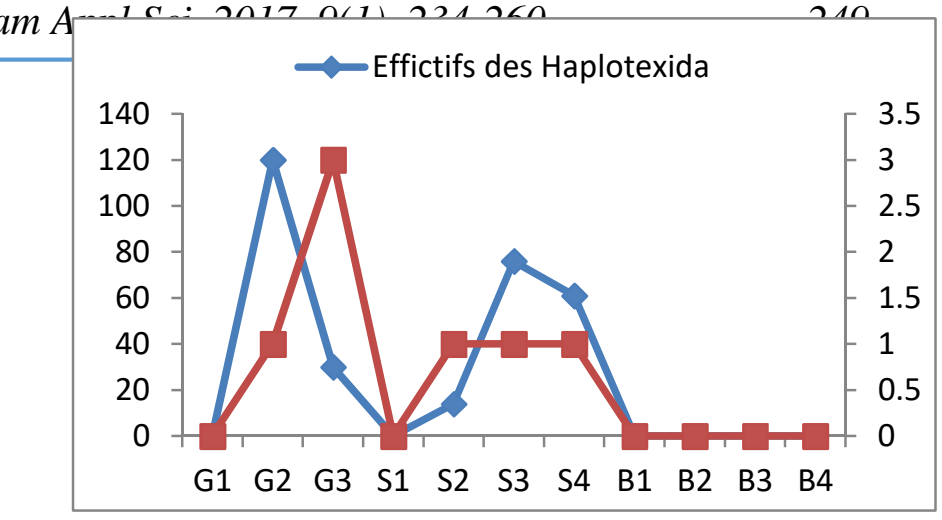

(B)

Fig. 5. Abondance (A) répartition longitudinale (B) des Haplotexida

\section{Les Odonates}

Les Odonates seraient le meilleur exemple puisqu'elles sont au sommet de la chaine trophique des macro-invertébrés benthiques ce qui permet de tirer des informations relatives à la richesse faunistique des milieux colonisés et permettraient par conséquent de détecter les infimes perturbations de l'écosystème. Par ailleurs, les Odonates sont généralement utilisées dans des études phytoécologiques, notamment pour caractériser les macrophytes, vu la relation trophique qui les relient. Ainsi, chaque changement de la végétation se répercuterait sur la composition et l'effectif de l'odonatofaune [19]. Dans le sous bassin versant de Boumerzoug, les Odonates se présente sous forme larvaire, l'abondance de ce groupe est assez diversifiée : 239 individus (soit 3,24\%) de la faune totale. Trois familles ont été récoltées : Caenagrionidae (236 individus), Libellulidae(2 individus), la troisième famille se présente sous forme (1'oeuf). Les larves Récoltés se répartissent en 3 genres :Caenagion,Enallagna et Orthetrum. La distribution longitudinale le long de trois oueds, les Odonates ont été relevés dansles stations (G3,S1,S3,S4,B1 et B2), avec une abondance maximale dans la station B1 (120 individus ) (Fig.6) : ce dernier résultat, se traduit par la vitesse du courant lente, par les température assez élevée des eaux et par une végétation assez développé dans ce tronçon de l'oued. 


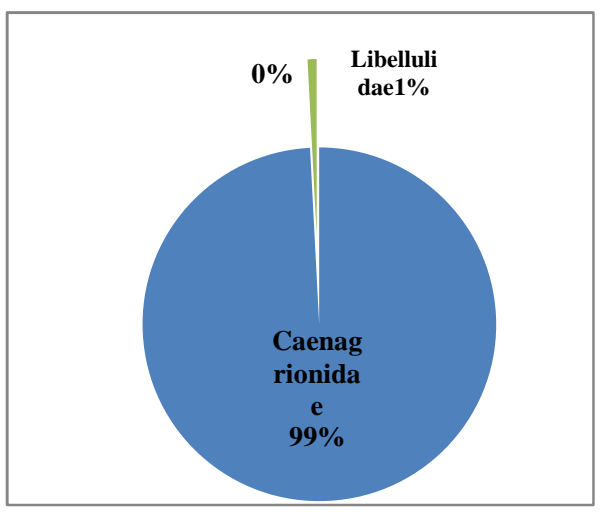

(A)

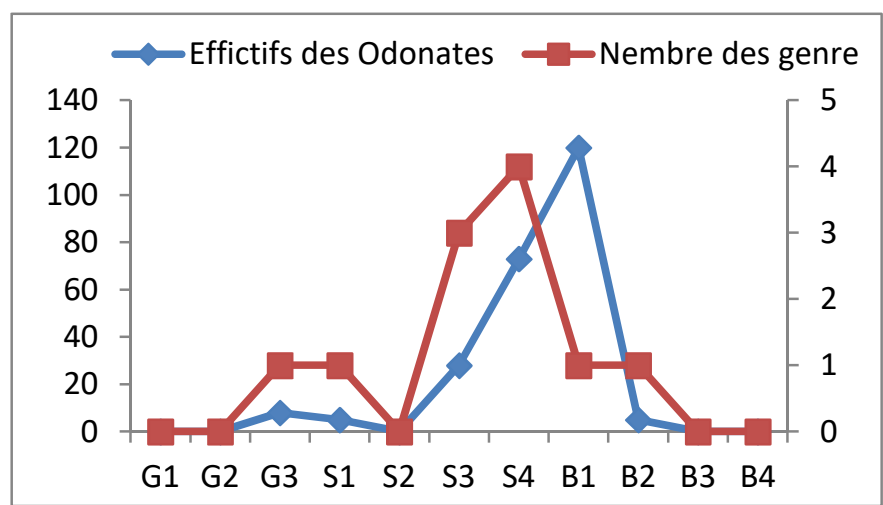

(B)

Fig.6. Abondance (A) répartition longitudinale (B) Les Odonates

\section{Podocopida}

Les Podocopida, avec 206 individus (soit $2,79 \%$ de la faune totale), sont représentés par une seule famille Candonidaeet deux genresCryptocandona et Candona. La répartition longitudinale des Podocopida(Fig.7), montre qu'ils sont représentés seulement dans les stations (S1, B1 et B2) .Candonacandia est l'espèce la plus dominante et surtout dans la

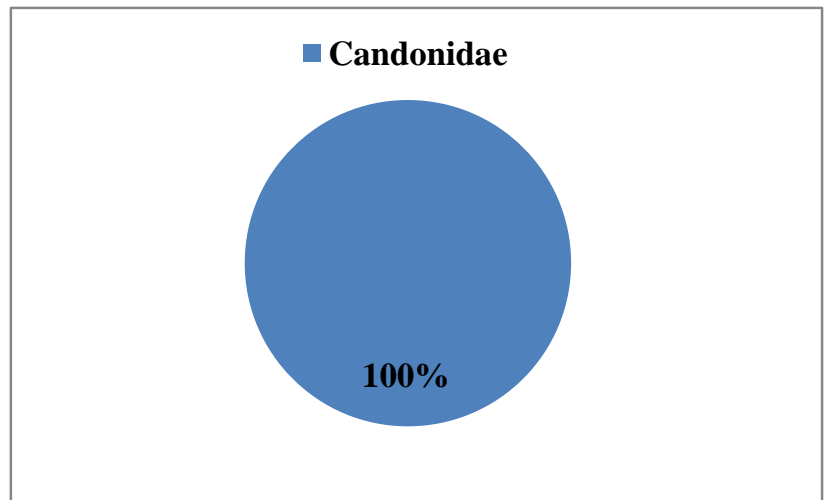

station B1 avec 135 individus.

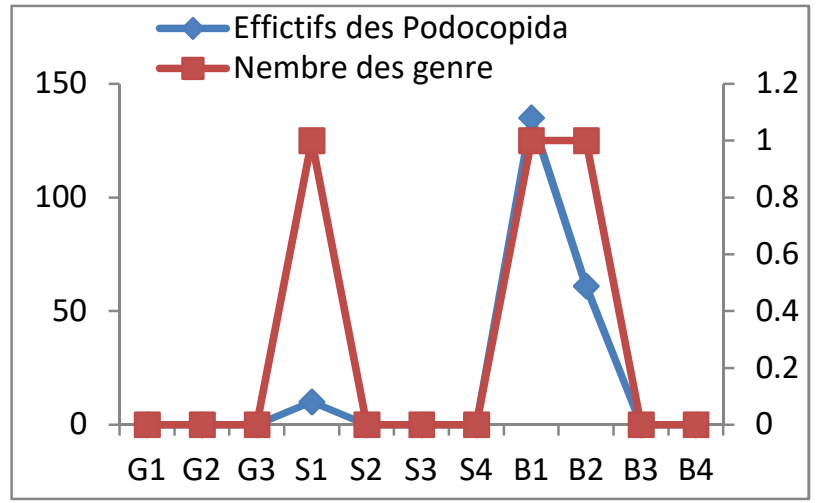

(A)

(B)

Fig. 7. Abondance(A) et répartition longitudinale (B) des Podocopida

\section{Pulomonea}

Les Pulomoneades cours d'eau étudiés relativement peu fréquents et peu abondants par rapport aux Odonates, HaplexidatesetPodocopida. Nous avons Récoltés 205 individus, soit 2,78\% de la faune totale, repartis en 8 familles et 33 genres. Les Physidaesont dominant avec 80 individus (37\%), et représente par deux genres PhysaetAplexa.Lesautre familles sont 
moins représentées (Fig.8). La répartition longitudinale des Pulomonea montre que leur prolifération semble plus importante surtout dans les stations (S1, B1et B2) dont les espèces Physasp et Physellagyrina sont les plus dominantes et occupent toutes les eaux de sous bassin.

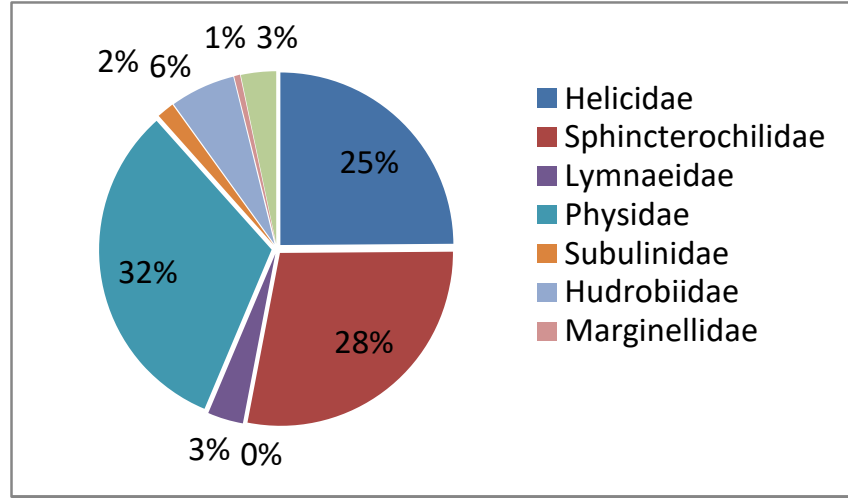

(A)

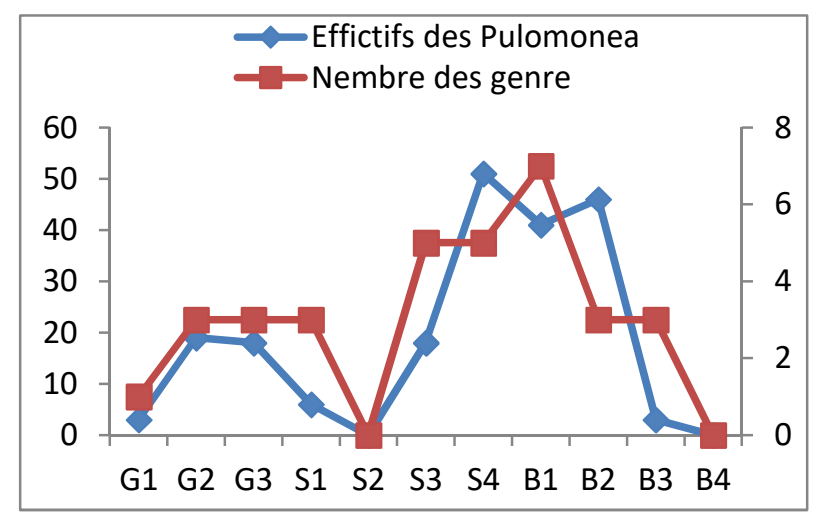

(B)

Fig.8. Abondance(A) et répartition longitudinale (B) des Pulomonea

\section{Ephéméroptères}

Les Ephéméroptères constituent l'ordre d'insectes le plus primitif parmi les insectes ailés. Connues depuis le Carbonifère, présentes et intimement liées à la vie aquatique, les éphémères sont très diversifiées à la fois sur le plan des habitats colonisés par les larves (eaux courants, eaux stagnantes), des relations qu'elles entretiennent avec le substrat (comportement fouisseur, de reptation ou nageur) ainsi qu'au niveau des comportements alimentaires (broyeur détritivore, racleur de substrat ou filtreur) [20]. 173 individus répartis en 2 genres appartenant à 2familles (Baetidae, Caenidae), ils représentent 2,34\% de la faune totale (figure9). La répartition en individus des Ephéméroptères dans les différentes stations étudiées met en évidence une diminution de nombre d'individus de stations (S3, B1) et une augmentation de stations (G2, S1, S2, G4). Ce résultat est lié à la nature de ces habitats caractérisés par des températures relativement élevées et un substrat hétérogène riche en matière organique, offrant des conditions fortement favorable d'une faune plus abondant surtout les Baetidae qui sont des éphéméroptères qui supportent la pollution [21]. 


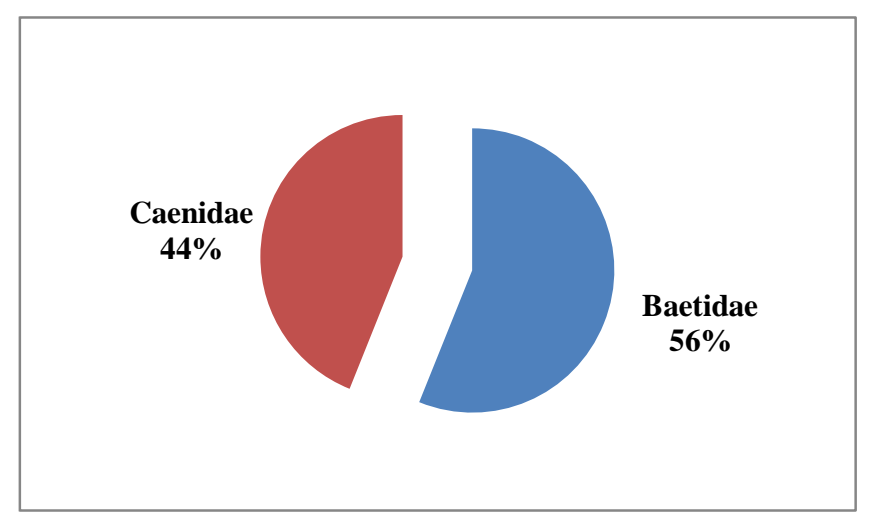

(A)

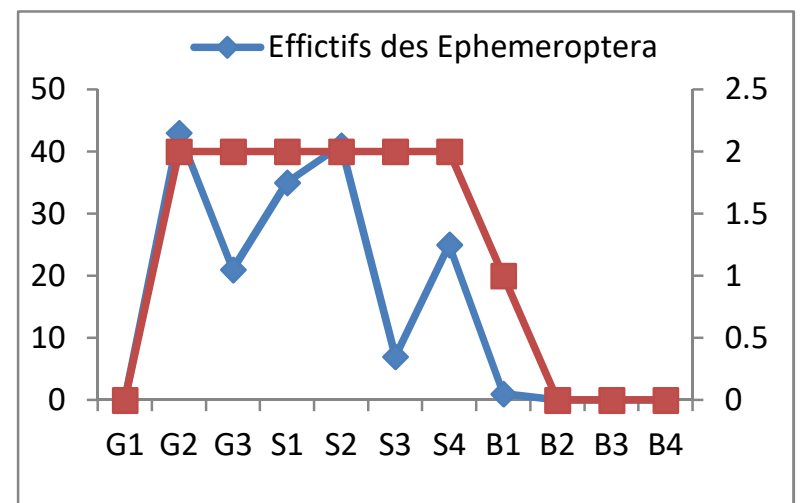

(B)

Fig.9 .Abondance (A) et répartition longitudinale (B) de Les Ephéméroptères

\section{Nematomorpha}

Ils sont représentés par 150 individus, soit 2,03\% de la faune totale récoltée .Ils sont Représentes par une seule famille et par une seule espèce Nematomorphasp., ces invertébrés sont collectés seulement dans la station G2 (Fig.10).

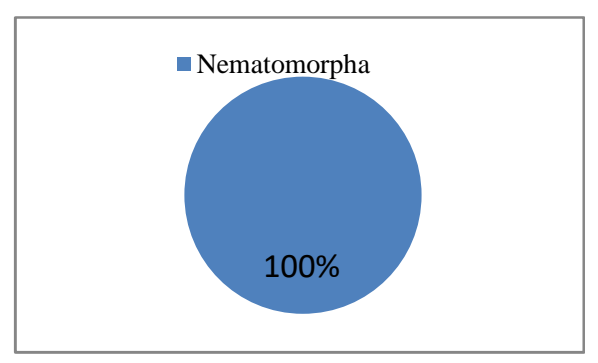

(A)

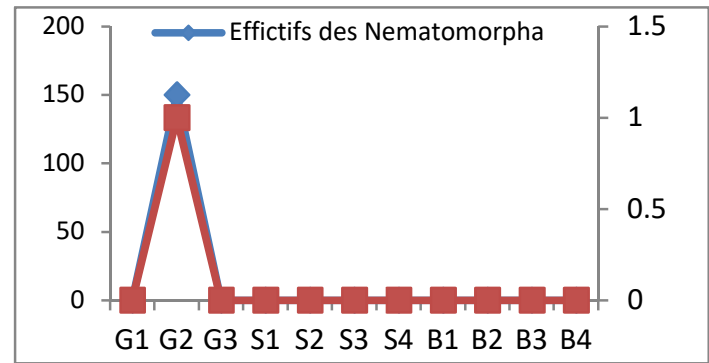

(B)

Fig.10. Abondance(A) et répartition longitudinale (B) des Nematomorpha

\section{Eulamellibranches}

Les Eulumellibranches rencontrés dans les cours d'eau étudiés représentent 1,81\% (134 individus) de faune récoltée. Ils sont constitués par une seule famille (Pisidae) et une seule espèce Pisidiumsp. Ils estcollecté seulement dans les stations B1 (20individus) et station B2 (114 individus) (Fig.11). 


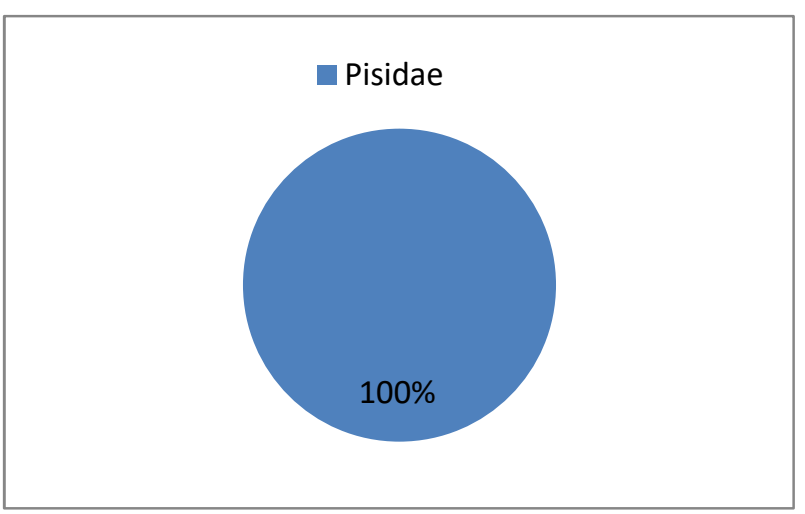

(A)

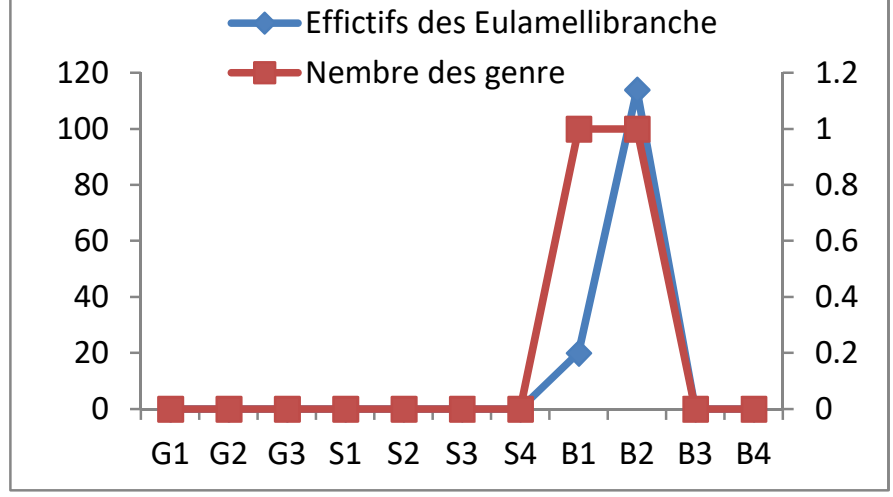

(B)

Fig.11. Abondance(A) et répartition longitudinale (B) desEulamellibranches

\section{Coléoptères et Amphipoda}

Les coléoptères et les Amphipodes sont faiblement représentés. Ils constituent respectivement 70 individus (soit $0,95 \%$ ) et 89 individus (soit $1,20 \%$ ) de la faune totale.

Les Coléoptères vivent pratiquement dans tous les biotopes, excepté les milieux polaires et océaniques. La biologie des espèces est très diverse, avec des exigences écologiques parfois très strictes qui en font d'excellents bio-indicateur cas des espèces saproxyliques ou des Scarabéidés,coprophages [22]. Si l'on considéré la richesse taxonomique au niveau de chaque famille, les Chrysomelidae et Dytixidae sont les mieux représentée. Elles comptent chacune respectivement 5et 3 genres. Les autres familles sont représentées que par un ou deux genres. L'analyse de la distribution longitudinale des Coléoptères (Fig.12). La richesse la plus élevée est observée dans les stations (G2, S4 et B1) respectivement 10,5et 5taxons et des stations (G1, G3, S3, S2 et B2) respectivement 4, 4, 3, 1et 1 taxons. Ceci peut s'expliquer par le fait que les biotopes de ces stations offrent une plus grande variété de niche écologique, hétérogénéité du substrat et de la végétation.

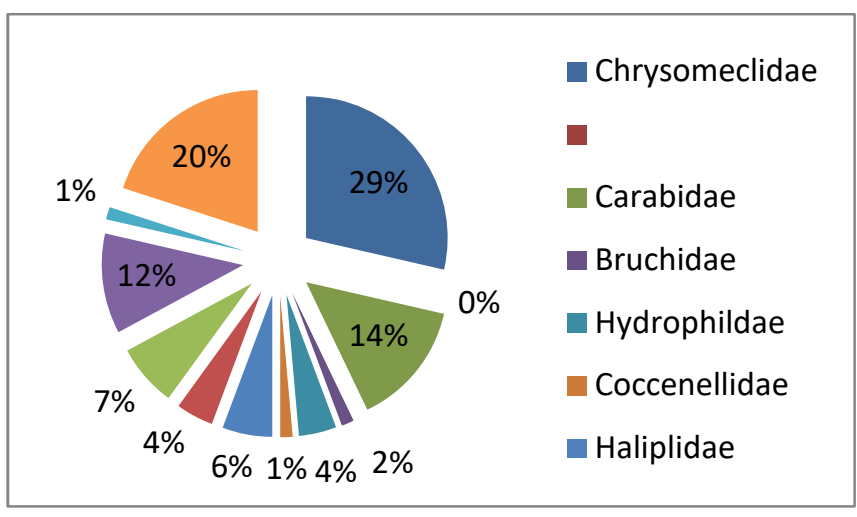

(A)

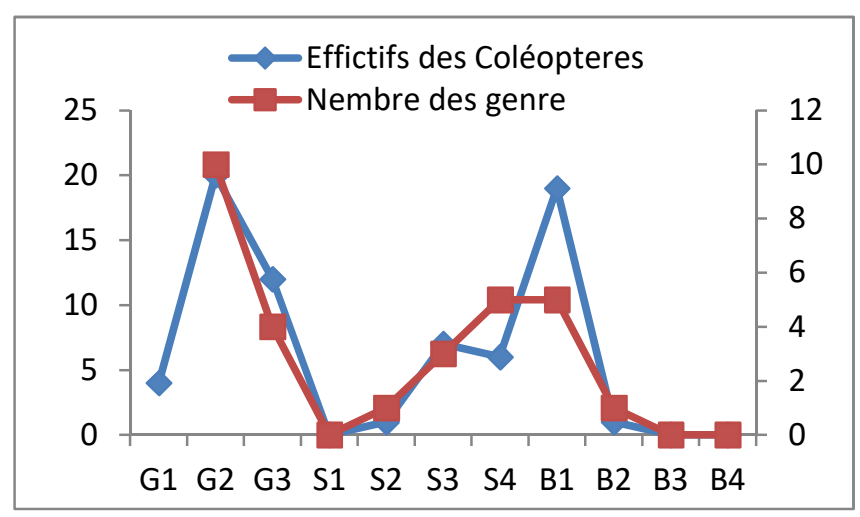

(B)

Fig.12. Abondance(A) et répartition longitudinale (B) des Coléoptères 


\section{Les Amphipodes}

Les Amphipodes sont représentés seulement par 89individus, soit 1,2\% de la faune totale. Ils sont représentés par une seule famille (Gammaridae) et par un seul genre (Gammarus). Ce groupe est collecté dans les stations (G3, S1, S2, S4, B1 et B2) avec un effectif important dans la station B2 (56individus) (Fig.13).De façon générale les Gammares sont grégaires et colonisent la plupart des cours ou étendues d'eaux rapides et froides de montagne ou rivières calmes et étangs de plaine, l'essentiel étant que l'eau soit suffisamment oxygénée, la température et la teneur en calcium de l'eau étant également des facteurs abiotiques importants. Les colonies se développent dans les herbiers qui bordent les cours d'eau, mais aussi entre et sous les pierres présentes sur le fond, et il n'est pas rare d'en trouver hors de l'eau à quelques centimètres du rivage dans des endroits humides sous les pierres [23].

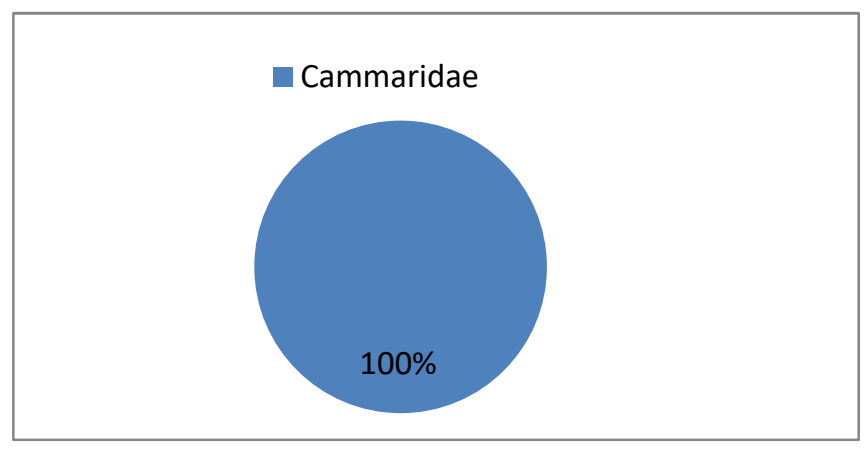

(A)

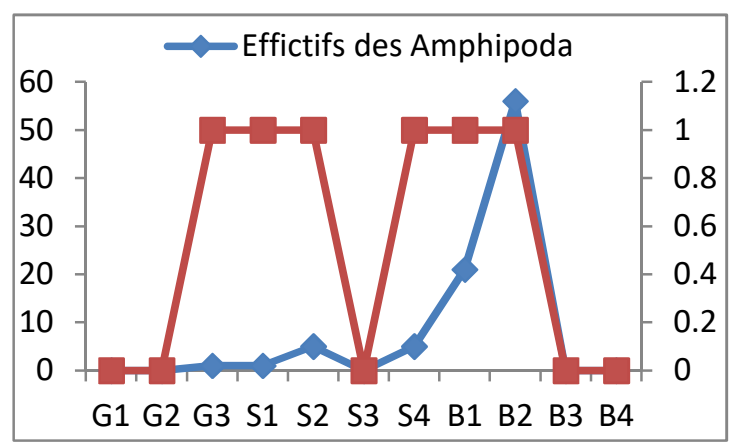

(B)

Fig.13. Abondance (A) et répartition longitudinale (B) des Amphipodes

\section{Les autres Ordres}

Les autres Ordres dans la présente étude sont représentés en très faibles proportions (Tab.1). En effet, la prospection des toutes stations nous a permis de récolter seulement 34individus(Hymenoptera),25individus(Archynchobdellida),16individus(Diplostraca),9indivu us(Rhynchodbelliformes),4individus(Opisthopoa),3individus(Aranea), 1individus(Dermaptea), 1'individus (Arcellinida). 
Table.1. l'Abondance et quantitative des Ordres qui sont faiblement représentés

\begin{tabular}{llll}
\hline Ordre & $\begin{array}{l}\text { Nombre } \\
\text { d'individus }\end{array}$ & $\begin{array}{l}\% \text { de la faune } \\
\text { totale }\end{array}$ & \\
\hline Hymenoptera & 34 & 0,46 & SaufS1,B3 \\
Archynchobdellida & 25 & 0,33 & $\mathrm{~S} 1, \mathrm{~S} 4$ \\
Diplostraca & 16 & 0,21 & $\mathrm{~S} 1, \mathrm{~B} 1$ \\
Rhynchodbelliformes & 9 & 0,12 & $\mathrm{~S} 3, \mathrm{~S} 4$ \\
Opisthopoa & 4 & 0,05 & $\mathrm{~S} 1, \mathrm{~S} 4$ \\
Aranea & 3 & 0,04 & $\mathrm{G} 2, \mathrm{~B} 1$ \\
Dermaptea & 1 & 0,01 & $\mathrm{~S} 3$ \\
\hline \hline Arcellinida & 1 & 0,01 & $\mathrm{~S} 4$ \\
\hline
\end{tabular}

\subsection{Analyse de la structure de peuplement par calcule de l'indice biologique Global Normalise (IBGN)}

Pour évaluer la qualité d'un cours d'eau, plusieurs méthodes dites «biologiques » sont utilisées. Une de ces méthodes, L'IBGN, consiste à analyser la présence des différentes familles de macro-invertébrés benthiques peuplant le fond des cours d'eau. L'IBGN (indice Biologique Globale Normalisé) est normalisé depuis le 20décembre 1992par décision de la direction général de L'AFNOR (Agence Française de Normalisation).Comme tout écosystèmes, les cours d'eau peuvent être soumis à diverses pollution ou altérations, qu'elles soient chimiques, physiques ou biologiques. En d'autre termes, les rivières sont menacées et l'existence de ces altérations aura pour conséquence de détériorer la qualité de l'écosystème (diminution du nombre de niches écologiques, diminution de la quantité d'oxygène dissous...).Ainsi, plus un milieu sera riche en terme de diversité taxonomique et plus la qualité du milieu sera bonne (stabilité et complexité des relations trophiques dans le cours d'eau). L'indice Biologique Global Normalisé est un moyen de rendre compte de cette richesse biologique dans une rivière, et cela dans le but de tirer des conclusions concernant la qualité du cours d'eau (qualité de l'eau et du biotope). L'IBGN tend à faire une étude plus compléte de la qualité de écosystème riviere.La présence des différents taxons de macroinvertebres benthique sur divers substrats pourra donner des renseignements à la fois sur la qualité de l'eau (polluosensibilité) mais aussi sur la qualité du milieu (diversité taxonomique). Ainis, il sera possible à la fin de l'étude d'attribuer une note globale du cours d'eau indiquant 
la qualité de l'écosystème pour comprendre quelles sont les altérations (physiques ou chimique) qui interviennent [11]. Les fluctuations de L'IBGN entre les stations dont mis en évidence 2 classes de qualité (Tab.2) :

$\checkmark$ Qualité médiocre avec une pollution importante pour les stations (G1.G2.G3.S1.S2.S3.S4 et B1un nombre de taxons réduit respectivement (17.18.19.17.19.19 et 27denombres .19) et un G1 très bas (1). Le GI(1) (Chironomidae) appartient aux groupes les plus polluo-résistants.

$\checkmark$ Par ailleurs les stations (B2.B3 et B4) dont L'IBGN ne dépasse pas 4 ont des eaux appartenant à la classe mauvaise qualité. Ces faibles valeurs pourraient s'expliquer par des faibles valeurs de groupes indicateurs et de la variété taxonomique dans ces stations résultat de la dégradation de la qualité des eaux dans ces stations par l'évacuation directe des rejets des eaux usées dans le milieu récepteurs.

Table. 2. Valeurs moyennes de l'indice biologie global normalisé au niveau des

\begin{tabular}{|c|c|c|c|c|c|c|c|c|c|}
\hline & $\begin{array}{l}\text { Effect } \\
\text { if } \\
\text { totale }\end{array}$ & $\begin{array}{c}\text { Variété } \\
\text { totale }\end{array}$ & $\begin{array}{c}\text { Classe de } \\
\text { variété }\end{array}$ & $\begin{array}{c}\text { Groupe } \\
\text { indicate } \\
\text { ur }\end{array}$ & $\begin{array}{c}\text { IBG } \\
\mathbf{N}\end{array}$ & $\begin{array}{l}\text { Qualité } \\
\text { d'eau }\end{array}$ & Couleur & $\begin{array}{c}\text { Polluti } \\
\text { on }\end{array}$ \\
\hline \multirow{11}{*}{ 参 } & G1 & 675 & 17 & 6 & 1 & 6 & Médiocre & \multirow{8}{*}{ 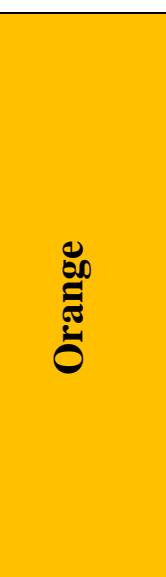 } & \multirow{8}{*}{ 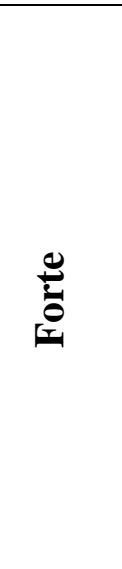 } \\
\hline & G2 & 558 & 18 & 6 & 1 & 6 & Médiocre & & \\
\hline & G3 & 574 & 19 & 6 & 1 & 6 & Médiocre & & \\
\hline & S1 & 156 & 17 & 6 & 1 & 6 & Médiocre & & \\
\hline & $\mathrm{S} 2$ & 833 & 19 & 6 & 1 & 6 & Médiocre & & \\
\hline & S3 & 1053 & 19 & 6 & 1 & 6 & Médiocre & & \\
\hline & S4 & 646 & 27 & 8 & 1 & 8 & Médiocre & & \\
\hline & B1 & 2472 & 19 & 6 & 1 & 6 & Médiocre & & \\
\hline & B2 & 285 & 9 & 3 & 2 & 4 & Mauvaise & \multirow{3}{*}{ Rouge } & \multirow{3}{*}{$\begin{array}{c}\text { Très } \\
\text { Forte }\end{array}$} \\
\hline & B3 & 102 & 5 & 2 & 1 & 2 & Mauvaise & & \\
\hline & B4 & 10 & 2 & 1 & 1 & 1 & Mauvaise & & \\
\hline
\end{tabular}

Stations étudiées

Variété totale : le nombre total de taxons identifiés (le nombre d'individus par taxon n'est pas pris en compte). 


\section{CONCLUSION}

La diversité taxonomique dépend directement de la nature du substrat, la vitesse du courant et de la densité du couvert végétale. Généralement. La richesse faunistique est plus faible dans les stationsB2, B3, B4 par rapport les autres stations. (Ces stations sont soumis aux pressions industrielles et urbanises de la cite BOUMERZOUG et la ville de JDOUR et cartier de CHELIA) ce qui montre un peuplement benthique perturbe. Les onze stations prospectées ont mis en évidence deux classes de qualité hydro-biologique : Une médiocre (IBGN, 6et8) dans les stations (G1, G2, G3, S1, S2, S3, S4 et B1) et mauvaise qualité (B2, B3 et B4) (IBGN, 1,2et4), caractérisées par une faible diversité taxonomique et la disparition des taxons les plus polluo-sensibles qui indique une probable altération de la qualité de l'eau (impact humain importants et rejets urbaines).

\section{REFERENCES}

[1] Gagneur J., Thomas A.G .B. Contribution à la connaissance des Ephéméroptères d'Algérie .Repartions et écologie ( $1^{\text {ere }}$ partie), (Insecta, Ephéméroptère). Bull. Soc. Hist. Nat., Toulouse, 124,1988, pp. :213-223.

[2] Lounaci A. les macro-invertébrés benthiques des cours d'eau de Kabylie : faunistique, écologie et répartition géographique. Congrès animal de la SZF, Parc Phoenix, Nice, 13-16 septembre 2011.

[3] Ferganie, Araba. Utilisation des macro-invertébrés benthiques comme bio indicateurs de pollution d'oued El Harrach. USTHB-FBS-4th international Congress of the populations \& Animal communities. « CIPCA4 »TACHIT (Bechar)-ALGERIA, 19-21 Novembre, 2013.

[4] Moisan J., Pelletier L. Protocole d'échantillonnage des macro-invertébrés Benthiques d'eau douce du Québec, cours d'eau peu profonds à substrat meuble, Direction du suivi de l'état de l'environnement, ministère du Développement durable, de l'environnement et des Parcs, ISBN :978-2-550-611660(PDF), 2011,39 pages.

[5] Benghorieb N., Siline R. Utilisation de Daphniamangna comme bio indicateur de qualité d'eau d'Oued Rhumel. Mémoire d'ingéniorat d'état en Ecologie .Univ. Constantine. 2012, $60 \mathrm{p}$.

[6] Benchalal W., Samraoui B. Caractérisation écologique et Biologique de l'odonatofaune de deux cours d'eau méditerranées : l'oued El Kébir et l'oued Bouaroug (Nord -Est de l'Algérie. Méditerranée n¹18.pp.19-27, 2012. 
[7] Geninb, Chauvinc, Menarde. Cours d'eau et indice biologiques, pollution méthodesIBGN : 222, (2003).

[8] Agence du Bassin. Hydrographique (ABH). Les Cahiers de l'Agence $N^{\circ}$ 8.Le Bassin du Késbir- Rhumel. Agence de Bassin Hydro-graphique Constantinois - SeybouseMellegue,Ministére des Ressources en Eau,42p., 2002.

[9] Southwoodt R.E. Habitat, The templet for ecological stratidies.Journal of Animal écologie 46: 337-365, 1977.

[10] Haouchine S. Recherches sur la faunistique et l'écologie des macro- invertébrés des cours d'eau de Kabylie .Thèse Magister, Univ.Tizi ouzou : 157(2011).

[11] Avesque C. et al. Pré-rapport sur les Indices Biologiques «Année2009 ».polytech Montpellier STE4. Promotion 2010. P64.

[12] Tachet H., Bournaud.M., Richoux Ph. et Usseglio-Polatera.Ph. Invertébrés d'eau douce : systématique, biologie, écologie, CNRSéditions, Paris : 587(2000).

[13] Khettar S., Haouchine N., Asselah B. Macro-invertébrés et Qualité biologique de Quelques cours d'eau du bassin versant de la Macta (ouest de L'Algérie).the $4^{\text {th }}$ Internationnal congress water, waste Environnement (EDE4) Agadir,Morocco,Décembre 18-20,2013.

[14] Sellam N., Vinolas A., Zouggaghe F., Moulai R. L'utilisation des Coléoptère,Ephéméroptère et Diptèra comme bio indicateurs de la qualité des eaux de quelques oueds en Algérie. Butlleti de la Institucio Catalana d'Historia Natural, 80 :47-56, 2016.

[15] Debbiche Zerguine K. Contribution à l'étude des Chironomidae (Diptera, Insecta) des mares temporaires de la Numidie Orientale. Aspect de Biologie, Ecologie et systématique. ThèseDoctorat. Universitéd'Annaba. Algérie .p319, 2010.

[16] Boulkenaffet F. Contribution à l'étude de la biodiversité des Phlébotones (Diptera :Psychodidae) et appréciation dela faune Culicidienne (Diptéra :Culicidae)dans la région de Skikda. Thèse deMagister.Université de Constantine.Algerie.p190, 2006.

[17] Boukhateb Y. Etude des Diptères Simuliidae de la haute Tafna (Nord-Ouest algérien).Mémoire de Master 2. Université de Tlemcen .p54, 2013. 
[18] MARTIN P., Ait Boughrous A. Guide taxonomique des oligochètes dulçaquicoles du Maghreb. P194, 2012.

[19] Limem Sellami E., Meurgey F., Barbouche N., Romdhane M.S. Odonates dans les principaux cours d'eau de parc national de L'Ichkeul (Tunisie). Entomologie Faunistique 68, p93-100, 2015.

[20] Zrelli S., Boulaabas Bejaoui M., Boumaiza M., Sartori M . Description et répartition de Potamanthus luteus Linnaeus 1767 (Ephéméroptèra,Potamanthidae) en Tunisie. Entomologie faunistique68, p213-218, 2015.

[21] Ben Moussa A., Chahlaoui A., Rour E., Chahboune M. Diversité taxonomique et structure de la macrofaune benthique des eaux superficielles de l'oued Khoumane. Moulay Idriss Zerhoun, Maroc.j.Mater.Environ.Sci.5(1), 183-198, 2004.

[22] Bouklihacene S. Bio-écologie des Coléoptères (Arthropodes-Insecte) du marais salé l'embouchure de la Tafna (Tlemcen). Thèse Doctorat. Université de Tlemcen.Algerie.p142, 2012.

[23]Graf F. Les Amphipodes de Bourgogue. Rev. Sci. Bourgogne-Nature (6) ,14-23, 2007. 


\section{How to cite this article:}

Bekhouche N, Marniche F, Ouldjaoui A.Contribution to the study of the biodiversity of benthic invertebrates and the biological quality of some rivers in the watershed boumerzoug (east of algeria). J. Fundam. Appl. Sci., 2017, 9(1), 234-260. 